\title{
A systematic review of the quality and scope of decision modelling studies in child oral health research
}

Greig D. Taylor ${ }^{1,2^{*}}$, Katherine Carr ${ }^{1}$, Helen J. Rogers ${ }^{1,2}$ and Chris R. Vernazza ${ }^{1,2}$

\begin{abstract}
Background: Decision analytic models are often used in economic evaluations to estimate long-term costs and effects of treatment which span beyond the time-frame of a clinical trial, therefore providing a better understanding of the long-term implications of decisions that conventional trial-based economic evaluations fail to provide. This is particularly relevant for considering oral health interventions in children as treatments may affect adult oral health. However, in the field of child oral health there has not been an evaluation of the quality and scope of decision analytical models which extend into adulthood. The aim of this review is to examine the scope and quality of decision modelling studies, with horizons extending into adulthood, within the field of child oral health.

Methods: The following databases were searched: NHS Economic Evaluation Database (CRD York), MEDLINE, EMBASE, CINAHL, Web of Science, Scopus, the Cochrane Library and Econlit. Full economic evaluations, in the field of child oral health, published after 1997 which included a decision model with a horizon that extended beyond the age of 18 years old were included. Included studies were appraised against the Drummond checklist and the Consolidated Health Economic Evaluation Reporting Standards by calibrated reviewers.

Results: Four hundred studies were identified, of which nine met the inclusion criteria. Of the nine, eight were cost-effectiveness models. The majority focussed on the prevention or management of dental caries. The mean percentage of applicable Drummond checklist criteria met by the studies in this review was $82 \%$ (median $=85 \%$, range $=54-100 \%$ ). Discounting of costs and performing an incremental analysis were noted as key methodological weaknesses. The mean percentage of applicable CHEERS criteria met by each study was $82 \%$ (median $=87 \%$, range $=32-96 \%$ ). Justifying the type of model, analytical methods used, and sources of funding were most commonly unreported.
\end{abstract}

Conclusions: There is a paucity of decision analytical models in the field of child oral health. Most of those that are available are of high methodological and reporting quality.

Keywords: Health economics, Children, Decision analytical modelling

*Correspondence: greig.taylor@newcastle.ac.uk

1 School of Dental Sciences, Faculty of Medical Sciences, Newcastle

University, Newcastle upon Tyne, UK

Full list of author information is available at the end of the article

\begin{abstract}
Background
Dental disease is prevalent and carries a high burden amongst children worldwide. Untreated dental caries [1] and molar-incisor hypomineralisation $(\mathrm{MIH})[2]$ are the most common oral disease in children, with an estimate of $9 \%$ [3] and $13.1 \%$ [2] respectively of children presenting with these diseases globally. Dental disease may directly
\end{abstract}

(c) The Author(s) 2021. Open Access This article is licensed under a Creative Commons Attribution 4.0 International License, which permits use, sharing, adaptation, distribution and reproduction in any medium or format, as long as you give appropriate credit to the original author(s) and the source, provide a link to the Creative Commons licence, and indicate if changes were made. The images or other third party material in this article are included in the article's Creative Commons licence, unless indicated otherwise in a credit line to the material. If material is not included in the article's Creative Commons licence and your intended use is not permitted by statutory regulation or exceeds the permitted use, you will need to obtain permission directly from the copyright holder. To view a copy of this licence, visit http://creativecommons.org/licenses/by/4.0/. The Creative Commons Public Domain Dedication waiver (http://creativeco mmons.org/publicdomain/zero/1.0/) applies to the data made available in this article, unless otherwise stated in a credit line to the data. 
impact a child as they could experience pain [4-8], either in isolation, or manifest during sleeping, eating/drinking or carrying out daily activities $[6,9,10]$, or have aesthetics and societal implications [11, 12]. Similarly, indirect impacts such as problems with school attendance [13], disturbances to general growth and development [10] as well as placing a burden on the parents, siblings and the wider general society have been reported $[6,14]$. It is therefore important, given these direct and indirect impacts, to know how best to manage these dental conditions in children. There is a need for better evidence to synthesise how best to decide to treat these conditions in addition to considering how such decisions made in childhood will impact the patient over their lifetime [15].

For any given dental disease, a range of interventions can be offered to manage the problem and reduce the impact the disease has on the child. Often different treatment options have different costs and effects associated with them and choosing which to provide can be determined by efficiency (i.e. which intervention offers the most benefit for the least cost or the most favourable cost:benefit ratio). This can be assessed using an economic evaluation, the results of which can support decision makers to maximising benefits from limited healthcare resources [16, 17]. Economic evaluations can be run alongside a clinical trial [18]. However, clinical trials have a finite time span (follow up period) and may only compare two mutually exclusive treatment options, where in reality, several may exist. In addition, trial-based economic evaluations use individual patient data, meaning that valid evidence from other trials or meta-analyses may be ignored when a judgement on the most efficient option needs to be made. Therefore using data gathered from a trial is unlikely to capture long-term costs and benefits of alternative interventions [16]. Decision analytic models (DAM) offer a way to model for long-term costs and benefits and can also synthesize evidence from similar trials to improve the robustness of the evaluation. This is hugely beneficial in decisions involving a child's care as it helps establish the most efficient option over the lifetime of the individual [19].

Previous systematic reviews of economic evaluations in dentistry have either focused on specific areas of dental disease [20-23] or economic evaluation methodologies [24, 25]. For example, Qu et al. [24], reported the methodological quality of DAMs studies involving interventions to manage dental caries in children and adults. However, the scope of this review was only specific to dental caries interventions, included adult patients and did not appraise the quality of reporting [24]. Rogers et al. [26] was the first systematic review to evaluate the scope and quality of economic evaluations in child oral health but excluded modelling studies that extended into adulthood. Excluding these studies meant they could focus on the benefits gained during childhood. However, the problem with this approach was that it precluded analysis of studies assessing interventions with benefits into adulthood. The aim of this study is to report a systematic review, considering both the scope and quality, of decision modelling studies within the field of child oral health which extend the time horizon beyond the age of 18.

\section{Methods \\ Protocol and registration}

The study protocol was registered with the Prospective Register of Systematic Reviews (PROSPEROCRD42020166717), and it followed the recommendations of the Preferred Reporting Items for Systematic Reviews and Meta-Analysis (PRISMA) statement for reporting.

\section{Search strategy}

A search strategy was developed (attached in Additional file 1). The included search terms related to the key concepts associated with the review question. These terms were combined and adapted to comply with the validated NHS CRD economic evaluation filters for MEDLINE, EMBASE and CINAHL. These were adapted, as required for the purposes of this search, for use in the remaining databases which are noted below.

On the 1st October 2020, a search of the published literature was undertaken, by one reviewer (HJR) in the following electronic databases: NHS Economic Evaluation Database (CRD York), MEDLINE, EMBASE, CINAHL, Web of Science, Scopus, the Cochrane Library and Econlit. Searches covered the period from commencement of each database system until the 1st October 2020.

The reference lists of included articles were examined to assess if additional relevant studies, that were not found during the database searches, should be included. Efforts were made to identify relevant unpublished 'grey' literature and conference proceedings through appropriate websites and databases such as OpenGrey. The EThOS database was also searched to identify relevant published UK theses.

\section{Eligibility criteria}

For studies to be included in this review, they had to meet the following inclusion criteria:

- Full economic evaluation in the field of child oral health

- Involved a decision model with a horizon that extended beyond the age of 18 years old

- Published after 1997 
No language restrictions were placed. It should be noted that cost-minimisation studies may not be universally considered as full economic evaluations, however, it was agreed by the research team to include them in this review. Similarly, it was agreed by the authors that only studies published after 1997 should be included in this review. This was due to the Drummond checklist [27], which was to be employed for this current review, only being available to researchers after this year.

Studies which used the lifetime of the tooth as a horizon were excluded. The reason for excluding these studies is that choosing such a horizon would not reflect the key differences in costs and consequences between interventions, e.g. if an evaluation compares filling and extraction of teeth, and the lifetime of the tooth was chosen as the horizon, once the tooth has been extracted the evaluation would cease yet there will still be benefits from the filling option that extend beyond this. Therefore, in these evaluations, comparing benefits obtained during childhood and adulthood from interventions carried in children cannot be fully assessed.

\section{Study selection and data extraction}

Search results were organised using Endnote ${ }^{\mathrm{TM}}$ X9. Duplicate articles were removed. Title and abstract screening against the eligibility criteria, was carried out independently by two reviewers (GDT \& KC), with any disagreement resolved by consensus. If necessary, any unresolved differences were resolved by a third reviewer (CRV).

Full texts were obtained for all titles that met these criteria. Two reviewers (GDT \& KC) assessed the full texts against the inclusion/exclusion criteria independently, with any disagreement resolved by consensus. If necessary, any unresolved differences were resolved by a third reviewer (CRV).

Two reviewers (GDT \& HJR) extracted data and assessed both the methodological quality, independently. A calibration exercise (using 3 papers) was conducted with all reviewers prior to commencement of data extraction and methodological/reporting quality appraisal. Any disagreement was resolved by consensus, and where needed, any unresolved differences were resolved by a third reviewer $(\mathrm{KC})$.

For each selected article, the following data, split into three sections, were extracted:

- Publication details publication year; author; country

- Study Characteristics oral health condition studied; nature of intervention

- Economic evaluation characteristics type of economic evaluation; perspective of the model; costing data sources; data source (primary/secondary data); horizon; model design; model input and parameter details; model outcome

\section{Quality assessment}

An assessment of methodological quality and reporting quality was undertaken. The most widely used checklist, the Drummond 10 item, 13 criteria checklist [27] (a simplified version of the 35-item Drummond version) was used to assess methodological quality. This checklist is recommended in the Cochrane Handbook for Systematic Reviews of Interventions [28].

To assess reporting quality, the Consolidated Health Economic Evaluation Reporting Standards (CHEERS) 24-item checklist was used [29]. Published in 2013, the CHEERS checklist aims to ensure consistent and transparent reporting of economic evaluations is carried out. The simplified Drummond, and CHEERS checklists have been previously used in published systematic reviews of economic evaluations assessing oral health interventions $[25,26]$. A score of $0,1,2$ was allocated for each criterion on the checklist:

Score 0: Criterion not met.

Score 1: Criterion met.

Score 2: Criterion not applicable.

Rogers et al. [26] reported cut-offs in their recent systematic review that appraised the quality and scope of economic evaluations in child oral health up to the age of 18 years old only. High, moderate and low cut-offs were assigned to each study based on their percentage applicability of Drummond [27] and CHEERS [29] criteria. The values used for these cut-offs were:

- Drummond Criteria: High > 50\%; Moderate 32-50\%; Low $<32 \%$ [26]

- CHEERS criteria: High > 83\%; Moderate 63-83\%; Low $<63 \%[26]$

It was agreed by the research team to use the same cutoffs for this review to allow a meaningful and direct comparison between the two studies.

\section{Statistical analysis}

Simple descriptive statistics, including inter- and intrarater reliability scores, were conducted on the extracted data and quality appraisal results using IBM $^{\circledR}$ SPSS $^{\circledR}$ Statistics v25. Meta-analyses were inappropriate given the diversity of interventions covered in addition to the aim of the review being to identify good practice and appraise quality. 


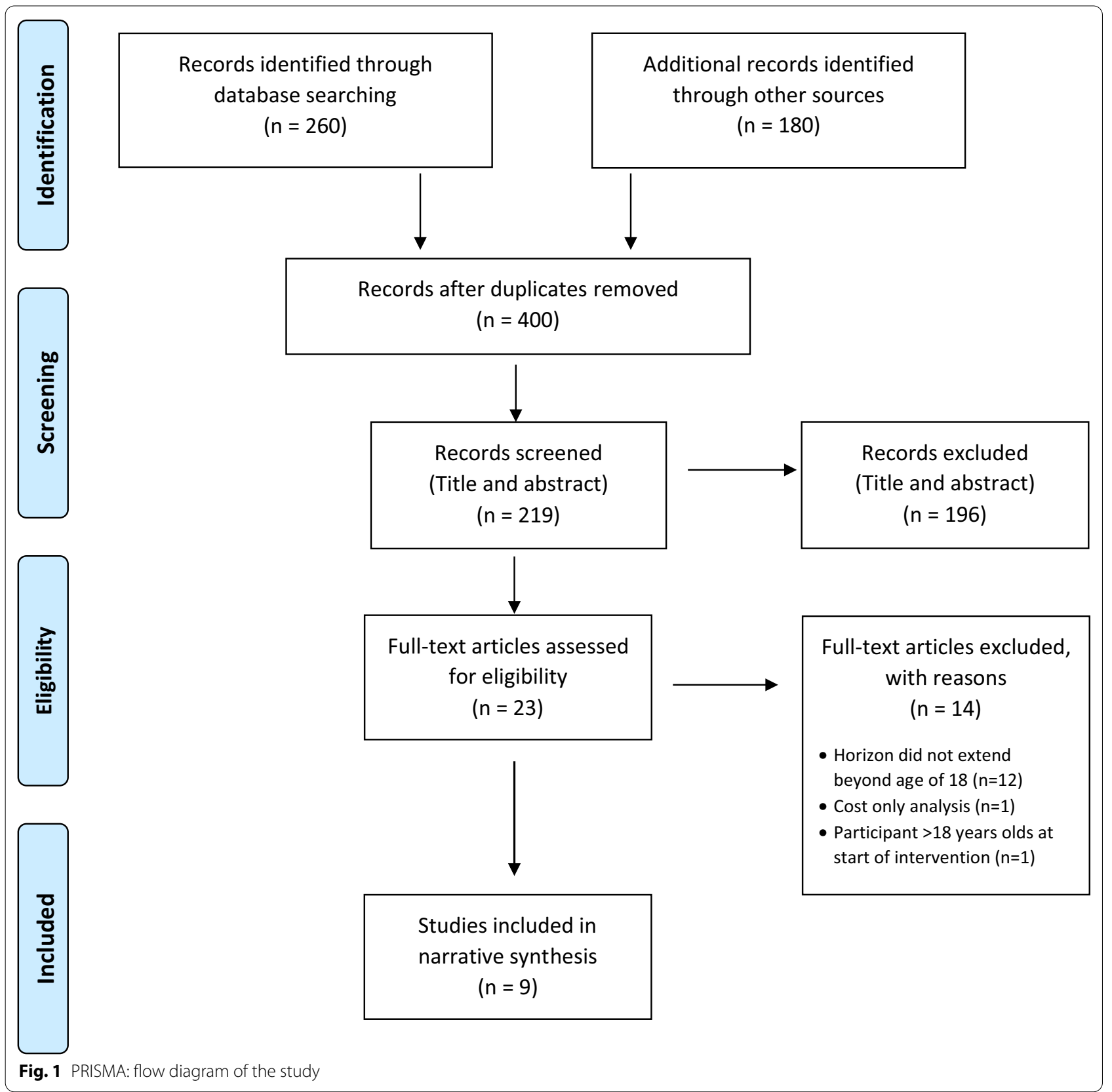

\section{Rater reliability}

Cohen's kappa (к) was calculated at 0.75 (93.2\% agreement) for overall inter-rater agreement for the Drummond checklist, and at 0.77 (91\% agreement) for the CHEERS checklist. These figures suggest substantial strength of agreement [30]. To determine intra-rater reliability, one of the included studies was randomly selected using an online random number generator and rereviewed four weeks later. Intra-rater agreement was 1.00 (100\% agreement) for both GT and HJR, when applying the Drummond checklist, and 0.71 (91.6\% agreement) for GT and 0.84 (95.8\% agreement) for HJR when using the CHEERS checklist.

\section{Results}

The search resulted in 440 articles and after the removal of duplicates, 400 articles were identified for title and abstract screening. Twenty-three papers were included for review of the full text. Fourteen were excluded because they did not meet the inclusion criteria. Nine 


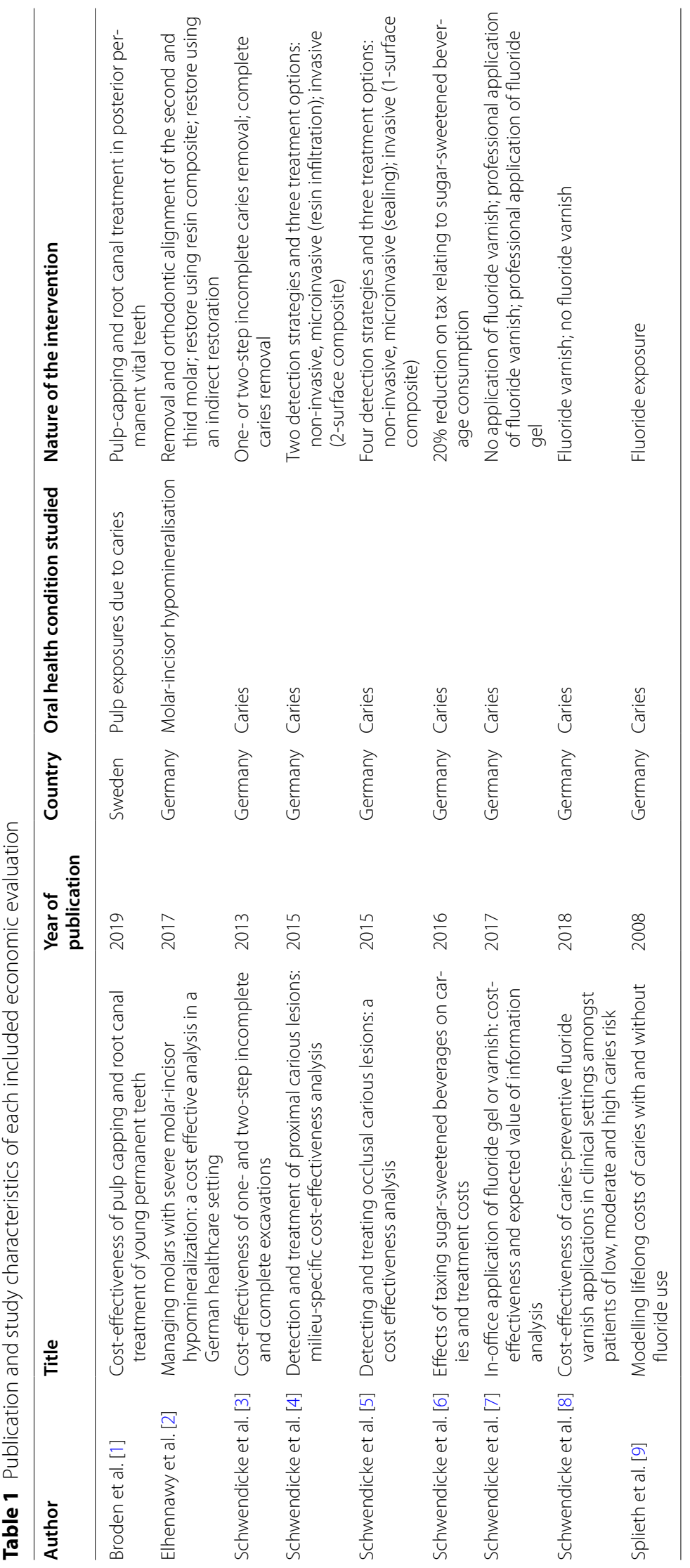




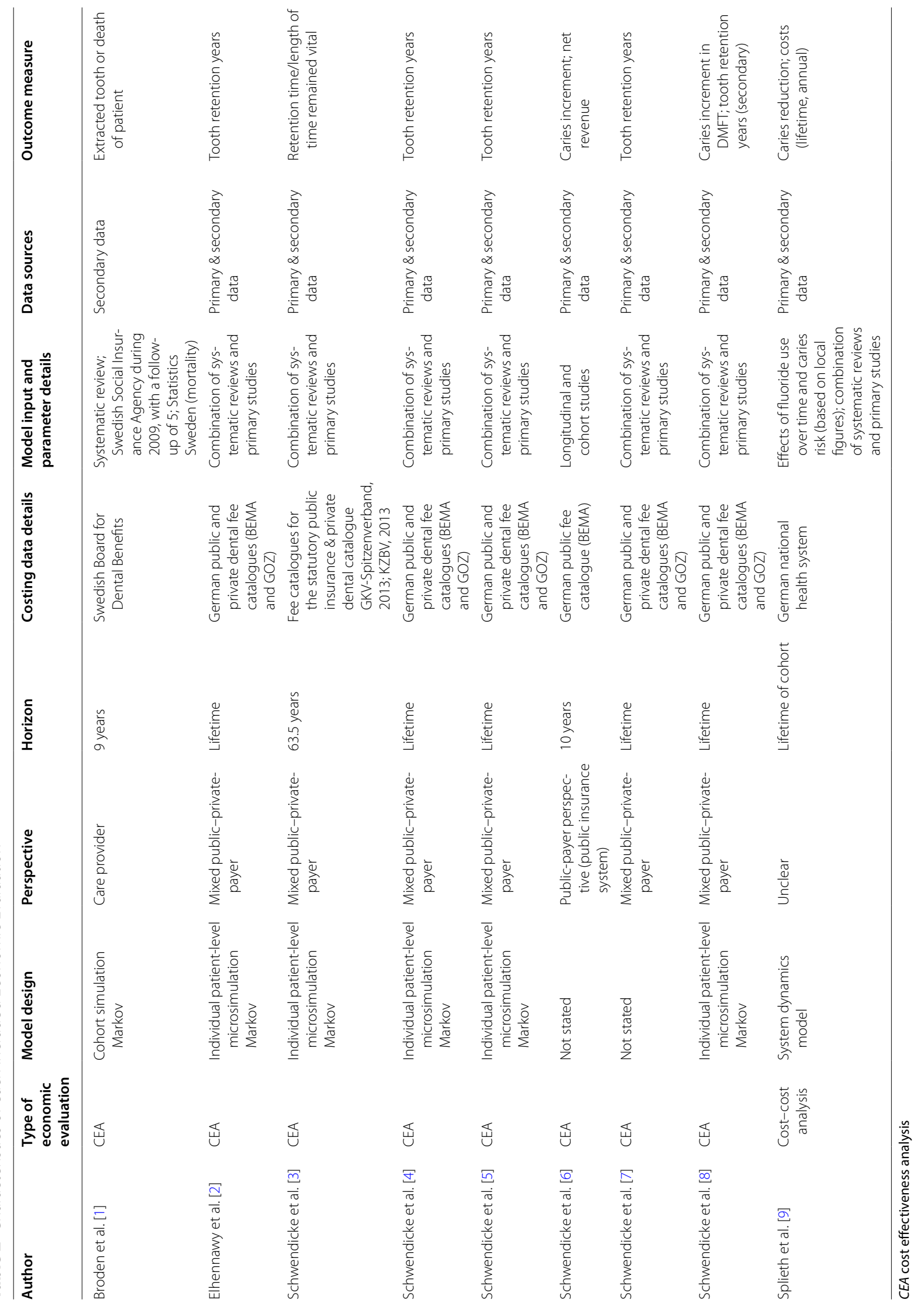


articles were included and underwent data extraction. Reference lists of included articles did not produce any further articles for inclusion. A summary of article selection is presented as a flowchart, based on PRISMA guidelines (Fig. 1). The publication and study characteristics and economic evaluation characteristics of the nine included studies are shown in Tables 1 and 2 respectively.

All nine studies included in the final analysis were written in the English language. All but one study undertook cost-effectiveness analyses $(n=8,89 \%)$. The remaining study reported a cost-cost analysis $(n=1,11 \%)$. The vast majority of studies were carried out in Germany $(n=8$, $89 \%$ ), with six being carried out by the same first author (66\%).

Most studies focused on the prevention or management of dental caries $(n=7,78 \%)$, with one study (11\%) relating to $\mathrm{MIH}$ and one relating to the caries increment as a result of a $20 \%$ tax on sugar-sweetened beverages (11\%). Just over half of the studies undertook an individual patient-level microsimulation Markov model $(\mathrm{n}=5,56 \%)$, one was a cohort simulation Markov model (11\%), one system dynamics model $(11 \%)$ and two did not clearly state their model design (22\%). Justification for the choice of model was not always provided. Two thirds of the studies used a lifetime horizon $(n=6,67 \%)$. One study used nine years (11\%), one ten years $(11 \%)$ and whilst the remaining paper used 63.5 years, detailed as the remaining life expectancy of a 15 -year old male simulated in their model (11\%).

The overall mean percentage of applicable criteria met by the studies in this review for the Drummond Checklist was $82 \%$ (median $=85 \%$, range $=54-100 \%$ ) and for the CHEERS checklist was $82 \%$ (median $=87 \%$, range $=32-96 \%$ ). Applicability scores and an assessment overall quality, based on pre-determined cut-offs [26], of for individual studies are shown in Table 3. In terms of quality, all nine studies were classified as high methodological quality in relation to the Drummond checklist. In comparison, seven studies were categorised as having high reporting quality, one moderate and one low quality in relation to the CHEERS checklist.

An overall breakdown of whether studies met each criterion of the Drummond and CHEERS checklists are shown in Tables 4 and 5 respectively. None of the studies reported on the measurement and valuation of preference-based outcomes, however, such outcome measures are more commonly applicable to cost-utility or costbenefit analyses, of which there were none identified in this review.

\section{Discussion}

To the author's knowledge, this is the first systematic review that has assessed the methodological and reporting quality of DAM studies, with horizons extending beyond childhood, within the field of child oral health. Limited examples of using DAMs exist in the field of child oral health; however, those that do exist are of relatively high methodological and reporting quality.

Using the high-, moderate- and low-level cut-offs of quality, as defined by Rogers et al. [26], the overall methodological quality was deemed to be high for all included studies in this review. In their study, Rogers et al. [26] reported that only $50 \%(n=23)$ of included studies were classified as high methodological quality. The median score for overall methodological quality in this review was $85 \%$, meaning that on average studies met 11 out of the 13 criterion of the Drummond Checklist [27]. This was much higher than the median score of $50 \%$ reported by Rogers et al. [26] It is unlikely that the threshold for the studies included in the Rogers et al. [26] review (studies were excluded if they involved decision models that extended into adulthood, or over a lifetime as to focus on the benefits from interventions gained solely during the childhood period) was the reason behind these differences. One possible explanation could be that the studies included in Rogers et al. [26] review were, in general, slightly older studies and therefore pre-date the Drummond checklist, which now commonly acts as a guide and an evaluation tool for economic evaluations. Alternatively, it could be that undertaking a decision analytical model that extends into adulthood, or over a lifetime, is methodologically more complex [19], and therefore in order to undertake the analysis many of the criteria listed in the Drummond checklist [27] were met as they were required to execute the model. Or, the number of authors to included studies ratio in each review may explain these differences. Rogers et al. [26] included a total of 46 studies led by 43 different authors. Whereas, of the nine studies included in this review, seven were carried out by the same German research group. It should be noted that the outcome of these DAMs is only pertinent to a German population, as the chosen perspective was that of a German mixed public-private-payer, and thus generalising these results outside of Germany is not permissible. However, this group's positive adherence to the guidance [31] and Drummond checklist [27], as shown by their high applicability of criterion met, could suggest why a high overall median value of methodological quality was obtained for this review. Interestingly, the median value for this review was consistent with that reported by Tonmykayakul et al. [23] for full economic evaluations in the wider field of dentistry, although, they did not specify which of the economic evaluations ran in conjunction 
Table 3 Drummond and CHEERS percentage applicability of each study

\begin{tabular}{lclll}
\hline Author & $\begin{array}{l}\text { \% applicable Drummond } \\
\text { criteria met }\end{array}$ & $\begin{array}{l}\text { Overall methodological } \\
\text { quality }\end{array}$ & $\begin{array}{l}\text { \% applicable CHEERS criteria } \\
\text { met }\end{array}$ & $\begin{array}{l}\text { Overall } \\
\text { reporting } \\
\text { quality }\end{array}$ \\
\hline Broden et al. [1] & 69 & High & 91 & High \\
Elhennawy et al. [2] & 100 & High & 87 & High \\
Schwendicke et al. [3] & 77 & High & 96 & High \\
Schwendicke et al. [4] & 69 & High & 86 & High \\
Schwendicke et al. [5] & 85 & High & 95 & High \\
Schwendicke et al. [6] & 85 & High & 86 & High \\
Schwendicke et al. [7] & 100 & High & 91 & Moderate \\
Schwendicke et al. [8] & 100 & High & 78 & Low \\
Splieth et al. [9] & 54 & High & 32 & \\
\hline
\end{tabular}

with a randomised control trial, or indeed included a decision analytical model, and therefore a meaningful comparison cannot be made.

Discounting of costs and performing an incremental analysis of the costs and outcomes were the most omitted criterion from studies included in this review. Future costs need to be discounted to reflect the amount spent or saved in the future should not weigh as heavily in decisions as those spent or saved today [19, 32]. This criterion is important for modelling studies in oral health interventions in children as dental disease is often a 'chronic' issue and the costs and outcomes associated with each decision will extend well into adulthood. The longer the horizon of the model, the more significant the difference is noted if discounting is not undertaken [19]. Therefore, as most studies included in this review used a lifetime horizon, by not discounting the costs could significantly impact the result.

Another criterion that was frequently absent was an incremental analysis of the outcomes and costs of alternatives. Omitting this analysis fails to provide the stakeholders, for whom the results from any modelling study will be essential to guide clinical and policy decisions, as to whether the additional costs generated by one alternative over another is compared to the additional benefits generated. It could be argued that an incremental analysis is a fundamental output of any given economic evaluation [16].

The overall reporting quality of economic evaluations included in this review was relatively high, with seven out of nine studies being in the high category. A median score of $87 \%$ against the CHEERS checklist was noted, which was similar to the median score of $83 \%$ reported by Rogers et al. [26]. Interestingly, the only study that was classified as low in this review was the oldest study, and similar observations were observed in the other review [26]. Reporting of economic evaluations of health interventions has been known to pose challenges, with various journals in the past offering their own advice [29]. The development of the CHEERS checklist in 2013 attempted to consolidate and update the various existing guidance into one useful resource [29]. This could explain why in both reviews the more recently published studies were noted to have a much higher reporting quality.

The worst performing criteria, in relation to the CHEERS checklist, were reporting the choice and justification of the chosen model, analytical methods used and sources of funding. Describing, but more importantly, justifying the choice of model, for example Markovmodel or decision tree, is an essential piece of information needed for any modelling study [33]. The type of model chosen should be driven by the clinical problem, with the model's structure being sufficiently complex to answer the proposed question in enough detail to ensure adequate external validity [31]. Without such information, the reader is unable to ascertain whether this is the best model to adequately address the decision problem in the given clinical or policy context. Similarly, a clear description of all analytical methods, including how to deal with uncertainties incorporated into the model, should be provided to ensure the results are valid [34]. Despite four studies failing to meet this criterion in this review, they did all provide some form of detail relating to their analytical approach. However, for a study to have met this criterion, for the purposes of this review, it was agreed between the reviewers that at least three of the five subsections had to have been reported. Finally, several studies failed to report their sources of funding, even if none existed. This declaration is important as non-disclosure could lead to a funding bias, and selective reporting of results [35].

The majority of the modelling studies in this review focussed on the prevention and/or management of dental caries. Similarly, Rogers et al. [26] noted that 42 (91\%) of 
Table 4 Breakdown of studies meeting each Drummond Criterion

\begin{tabular}{|c|c|c|c|c|}
\hline $\begin{array}{l}\text { Drummond } \\
\text { criterion }\end{array}$ & Summary of criterion & $\begin{array}{l}\text { Total studies } \\
\text { meeting criterion } \\
n=9\end{array}$ & $\begin{array}{l}\text { Total studies not } \\
\text { meeting criterion } \\
\mathrm{n}=9\end{array}$ & $\begin{array}{l}\text { Total studies where } \\
\text { criterion was not applicable } \\
n=9\end{array}$ \\
\hline 1 & Was a well-defined question posed in answerable form? & 9 & 0 & 0 \\
\hline 2 & $\begin{array}{l}\text { Was a comprehensive description of the competing alter- } \\
\text { natives given? }\end{array}$ & 6 & 3 & 0 \\
\hline 3 & $\begin{array}{l}\text { Was there evidence that the programme's effectiveness } \\
\text { had been established? }\end{array}$ & 7 & 2 & 0 \\
\hline 4 & $\begin{array}{l}\text { Were all the important and relevant outcomes and costs for } \\
\text { each alternative identified? }\end{array}$ & 6 & 3 & 0 \\
\hline $5 a$ & $\begin{array}{l}\text { Were outcomes measured accurately in appropriate units } \\
\text { prior to evaluation? }\end{array}$ & 9 & 0 & 0 \\
\hline $5 b$ & $\begin{array}{l}\text { Were costs measured accurately in appropriate units prior } \\
\text { to evaluation? }\end{array}$ & 9 & 0 & 0 \\
\hline $6 a$ & Were the outcomes valued credibly? & 9 & 0 & 0 \\
\hline $6 b$ & Were the costs valued credibly? & 9 & 0 & 0 \\
\hline $7 a$ & $\begin{array}{l}\text { Were outcomes adjusted for different times at which they } \\
\text { occurred? }\end{array}$ & 9 & 0 & 0 \\
\hline $7 b$ & $\begin{array}{l}\text { Were costs adjusted for different times at which they } \\
\text { occurred? }\end{array}$ & 3 & 6 & 0 \\
\hline 8 & $\begin{array}{l}\text { Was an incremental analysis of the outcomes and costs of } \\
\text { alternatives performed? }\end{array}$ & 5 & 4 & 0 \\
\hline 9 & $\begin{array}{l}\text { Was allowance made for uncertainty in the estimates of } \\
\text { costs and consequences? }\end{array}$ & 8 & 1 & 0 \\
\hline 10 & $\begin{array}{l}\text { Did the presentation and discussion of study results } \\
\text { include all of the issues that are of concern to users? }\end{array}$ & 8 & 1 & 0 \\
\hline
\end{tabular}

the studies included in their review was to deal with the prevention and/or management of caries. This is understandable given the prevalence of this dental condition amongst children [3]. Adopting a modelling approach, rather than a trial-based economic evaluation, is sensible for the management of dental caries in the permanent dentition as a randomised control trial is unlikely to determine the life-long impact on patients given the usual short-term follow-up of a trial. However, modelling approaches are not as important for management of dental caries in the primary dentition given their natural exfoliation occurs in childhood. A recent systematic review by Qu et al. [24] reported the application of DAM, and their methodological quality, in the context of dental caries across all age ranges. They included 25 studies and demonstrated that a variety of model structures were used, with a Markov model being most common [24]. They reported that the methodological quality of DAM in dental caries-related economic evaluations were unsatisfactory [24]. However the methodology of the review is unclear as some of the outcome measures listed in the included studies were not identified in the inclusion criteria.

Only one study in this review was not related to dental caries. Other dental conditions observed in children would benefit from modelling studies, for example management options for non-vital teeth with immature apices, management of anterior opacities due to MIH or the management of traumatic dental injuries. Although these conditions may not be as prevalent, these are just as impactful, and therefore this evidence found in this review supports the argument set out in Rogers et al. [26] that other conditions relating to child oral health should be priorities for future economic evaluation research.

All but one of the modelling studies included in this review conducted a cost-effectiveness analysis. Most of the studies either used tooth retention years or caries increment as their outcome measure, which are appropriate when being applied to modelling studies, as they are both able to capture long-term outcomes associated with the interventions. This predominance of cost-effectiveness analyses is consistent with previous systematic reviews addressing oral health interventions $[23,26]$. However other approaches to economic evaluation exist and should be considered in the field of child oral health. In the UK, the National Institute for Health Care and Excellence recommend cost-utility analyses, with results being reported in terms of the incremental cost per quality adjusted life year (QALY) gained [36]. Thus, allowing comparison across different areas of healthcare using a measure of health benefit that combines both quality of life and length of life into a single 
Table 5 Breakdown of studies meeting each CHEERS Criterion

\begin{tabular}{|c|c|c|c|c|}
\hline $\begin{array}{l}\text { CHEERS } \\
\text { Criterion }\end{array}$ & Summary of criterion & $\begin{array}{l}\text { Total studies meeting } \\
\text { criterion } \mathrm{n}=9\end{array}$ & $\begin{array}{l}\text { Total studies not meeting } \\
\text { criterion } n=9\end{array}$ & $\begin{array}{l}\text { Total studies where } \\
\text { criterion was not applicable } \\
\mathrm{n}=9\end{array}$ \\
\hline 1 & Title & 7 & 2 & 0 \\
\hline 2 & Abstract & 9 & 0 & 0 \\
\hline 3 & Background and objectives & 9 & 0 & 0 \\
\hline 4 & Target population and subgroups & 7 & 2 & 0 \\
\hline 5 & Setting and Location & 9 & 0 & 0 \\
\hline 6 & Study perspective & 9 & 0 & 0 \\
\hline 7 & Comparators & 8 & 1 & 0 \\
\hline 8 & Time horizon & 9 & 0 & 0 \\
\hline 9 & Discount rate & 8 & 1 & 0 \\
\hline 10 & Choice of health outcomes & 8 & 1 & 0 \\
\hline $11^{\mathrm{a}}$ & Measurement of effectiveness & 8 & 1 & 0 \\
\hline 12 & $\begin{array}{l}\text { Measurement and valuation of prefer- } \\
\text { ence-based outcomes }\end{array}$ & 0 & 0 & 9 \\
\hline $13^{\mathrm{a}}$ & Estimating resources and costs & 8 & 1 & 0 \\
\hline 14 & Currency, price date and conversion & 6 & 2 & 1 \\
\hline 15 & Choice of model & 4 & 5 & 0 \\
\hline 16 & Assumptions & 8 & 0 & 1 \\
\hline 17 & Analytical methods & 4 & 5 & 0 \\
\hline 18 & Study parameters & 8 & 1 & 0 \\
\hline 19 & Incremental costs and outcomes & 7 & 2 & 0 \\
\hline $20^{\mathrm{a}}$ & Characterising uncertainty & 7 & 2 & 0 \\
\hline 21 & Characterising heterogeneity & 3 & 1 & 5 \\
\hline 22 & $\begin{array}{l}\text { Study findings, limitations, generalisability } \\
\text { and current knowledge }\end{array}$ & 9 & 0 & 0 \\
\hline 23 & Source of funding & 4 & 5 & 0 \\
\hline 24 & Conflicts of interest & 7 & 2 & 0 \\
\hline
\end{tabular}

${ }^{\text {a }}$ Model-based criterion used

index [36]. Hettiarachchi et al. [25] did report that an increasing trend in the publication of cost-utility analyses in dentistry, however this was not observed in the present review as no modelling studies adopted a costutility analytical approach. This is not surprising, as commonly used tools to derive QALYs in children, have not been applied to oral health conditions other than caries. Foster-Page et al. [37] found that one tool, the CHU9D, was not sensitive enough to detect changes in caries status. However, it is worth noting that oral health specific instruments are emerging $[38,39]$. Alternatively, a cost-benefit analysis could be undertaken, where commensurate units (usually money) are used to value both costs and outcomes. Establishing willingness-to-pay values from preference elicitation methods, such as discrete choice experiments, can be used as a proxy for utility and therefore be used as an outcome measurement. These monetary values can be attached to health states in a model, in a similar way the units of outcomes for costeffectiveness and cost-utility models are. This approach could enable comparison across various dental disease profiles, which cannot always occur in cost-effectiveness analysis as the diseases studied may be measured in different units.

\section{Strengths and limitations}

This is the first systematic review that has addressed the scope and quality of economic evaluations that involve decision analytical models extending into adulthood in the field of child oral health. A strength of this review was the involvement of both paediatric dental and health economics expertise at each stage of the review. Furthermore, a calibration exercise was undertaken for data extraction and quality assessment, against both checklists, which was beneficial as shown by the intra- and inter-rater agreements scores. In addition, this review complemented the recent review by Rogers et al. [26], by overcoming their main limitation of excluding modelling studies which included decisions made about young people over the age of 18 -years. 
One acknowledged limitation was adopting the same cut-offs that were defined by Rogers et al. [26] These cut-offs were ascertained by using the median percentage, and interquartile range, of Drummond and CHEERS criteria met by the studies in their review, in an attempt to ensure that studies with a greater number of 'not applicable' criteria were not unfairly disadvantaged [26]. It could be argued that this review should have adopted a similar methodology, although, it was agreed by the research team that adopting these same cut-offs for high-, moderate- and low-quality studies would allow for a meaningful and direct comparison to a very similar and recent systematic review. The percentage for those studies classified as high in this review were all significantly higher than the predetermined cut-offs used. Incidentally, the number of criterion 'not applicable' to studies in this review was much less, comparatively, to that of Rogers et al. [26], and had cut-offs been created specifically for this review, the values would likely have all been higher. Nonetheless, this would have made very little difference to the overall quality assessment given the high percentages observed for each study, and each evaluation checklist, in this review.

\section{Conclusion}

There is a paucity of decision analytical models in the field of child oral health which span beyond the age of 18 , however the few that are available are of relatively high methodological and reporting quality. Decision analytical models can help, and should be used to, understand the implications of dental decisions made in children whereby the outcomes extend beyond 18 years old, and further work using this methodology in child oral health would be valuable.

\section{Abbreviations}

CHEERS: Consolidated Health Economic Evaluation Reporting Standards; DAM: Decision analytic models; MIH: Molar incisor hypomineralisation; PRISMA: Preferred Reporting Items for Systematic Reviews and Meta-Analysis; QALY: Quality adjusted life year.

\section{Supplementary Information}

The online version contains supplementary material available at https://doi. org/10.1186/s12903-021-01680-3.

Additional file 1. Search Strategy.

\section{Acknowledgements}

None.

\section{Authors' contributions}

GDT developed the protocol, reviewed and quality appraised the included papers, extracted the data, analysed the findings, and developed the manuscript. KC contributed to the protocol development, reviewed and quality appraised the included papers and extracted the data. HJR contributed to the protocol development, developed the search strategy, and conducted the database searches, reviewed and quality appraised the included papers and extracted the data. CRV contributed to protocol development and the reviewing process. All authors read and approved the manuscript.

\section{Funding}

Greig Taylor (NIHR300251), Katherine Carr (NIHR300683) and Helen Rogers (DRF-2016-09-094) are funded by a Doctoral Research Fellowship from the National Institute of Health Research (NIHR). The funding body was not involved in the design or conduct of this study, nor in the preparation of the manuscript. This article presents independent research funded by the NIHR. The views expressed are those of the authors and not necessarily those of the $\mathrm{NHS}$, the NIHR or the Department of Health and Social Care.

\section{Availability of data and materials}

All data generated or analysed during this study are included in this published article and its Additional file 1.

\section{Declarations}

Ethics approval and consent to participate

Ethical approval is not required for this study.

Consent for publication

Not applicable.

\section{Competing interests}

There are no competing interests.

\section{Author details}

${ }^{1}$ School of Dental Sciences, Faculty of Medical Sciences, Newcastle University, Newcastle upon Tyne, UK. ${ }^{2}$ Newcastle upon Tyne Hospital NHS Foundation Trust, Newcastle upon Tyne, UK.

Received: 26 February 2021 Accepted: 2 June 2021

Published online: 25 June 2021

\section{References}

1. Marcenes W, Kassebaum NJ, Bernabé E, Flaxman A, Naghavi M, Lopez A, et al. Global burden of oral conditions in 1990-2010: a systematic analysis. J Dent Res. 2013;92(7):592-7.

2. Schwendicke F, Elhennawy K, Reda S, Bekes K, Manton DJ, Krois J. Global burden of molar incisor hypomineralization. J Dent. 2018;68:10-8.

3. Kassebaum NJ, Smith AGC, Bernabe E, Fleming TD, Reynolds AE, Vos T, et al. Global, regional, and national prevalence, incidence, and disabilityadjusted life years for oral conditions for 195 countries, 1990-2015: a systematic analysis for the global burden of diseases, injuries, and risk factors. J Dent Res. 2017;96:380-7.

4. Selwitz RH, Ismail Al, Pitts NB. Dental caries. Lancet. 2007;369(9555):51-9.

5. Tsakos G, Hill K, Chadwick B, Anderson T. Children's dental health survey 2013, Report 1: attitudes, behaviours and children's dental health, England, Wales and Northern Ireland, 2015;2015.

6. Taylor GD, Scott T, Vernazza CR. Research Prize Category: impact of first permanent molars of poor prognosis: patient \& parent perspectives. Int J Paediatr Dent. 2018;28:2-3.

7. Rodd HD, Boissonade FM, Day PF. Pulpal status of hypomineralized permanent molars. Pediatr Dent. 2007:29:514-20.

8. Gilchrist F, Marshman Z, Deery C, Rodd HD. The impact of dental caries on children and young people: what they have to say? Int J Paediatr Dent. 2015:25(5):327-38.

9. Bernabé $E$, Tsakos $G$, Sheiham A. Intensity and extent of Oral Impacts on Daily Performances by type of self-perceived oral problems. Eur J Oral Sci. 2007;115:111-6.

10. Sheiham A. Dental caries affects body weight, growth and quality of life in pre-school children. Br Dent J. 2006;25(201):625-6. 
11. Hasmun N, Lawson J, Vettore MV, Elcock C, Zaitoun H, Rodd H. Change in oral health-related quality of life following minimally invasive aesthetic treatment for children with molar incisor hypomineralisation: a prospective study. Dent J [Internet]. 2018;6(4):61.

12. Porritt JM, Rodd HD, Baker SR. Quality of life impacts following childhood dento-alveolar trauma. Dent Traumatol. 2011;27:2-9.

13. Rebelo MAB, Rebelo Vieira JM, Pereira JV, Quadros LN, Vettore MV. Does oral health influence school performance and school attendance? A systematic review and meta-analysis. Int J Paediatr Dent. 2018.

14. Listl S, Galloway J, Mossey PA, Marcenes W. Global economic impact of dental diseases. J Dent Res. 2015;94(10):1355-61.

15. Taylor GD, Pearce KF, Vernazza CR. Management of compromised first permanent molars in children: cross-sectional analysis of attitudes of UK general dental practitioners and specialists in paediatric dentistry. Int J Paediatr Dent. 2019;29(3):267-80.

16. Drummond M, Sculpher M, Claxton K, Stoddart G, Torrance GW, editors. Methods for the economic evaluation of health care programmes. 4th ed. Oxford: Oxford University Press; 2015.

17. Eddama O, Coast J. A systematic review of the use of economic evaluation in local decision-making. Health Policy. 2008;86(2):129-41.

18. Rogers HJ, Freitas RD, Beeson MJ, Vernazza CR. Economic evaluations in paediatric dentistry clinical trials. Int J Paediatr Dent. 2020;31(S1):56-65.

19. Petrou S, Gray A. Economic evaluation using decision analytical modelling: design, conduct, analysis, and reporting. BMJ [Internet]. 2011;342:d1766.

20. Källestål C, Norlund A, Söder B, Nordenram G, Dahlgren H, Petersson $L G$, et al. Economic evaluation of dental caries prevention: a systematic review. Acta Odontol Scand. 2003;61(6):341-6.

21. Mariño RJ, Khan AR, Morgan M. Systematic review of publications on economic evaluations of caries prevention programs. Caries Res. 2013;47(4):265-72.

22. Mariño R, Ravisankar G, Zaror C. Quality appraisal of economic evaluations done on oral health preventive programs-A systematic review. J Public Health Dent. 2020;80:194-207.

23. Tonmukayakul U, Calache H, Clark R, Wasiak J, Faggion CM. Systematic review and quality appraisal of economic evaluation publications in dentistry. J Dent Res. 2015;94(10):1348-54.

24. Qu Z, Zhang S, Krauth C, Liu X. A systematic review of decision analytic modeling techniques for the economic evaluation of dental caries interventions. PLoS ONE [Internet]. 2019;14(5):e0216921.

25. Hettiarachchi RM, Kularatna S, Downes MJ, Byrnes J, Kroon J, Lalloo $R$, et al. The cost-effectiveness of oral health interventions: a systematic review of cost-utility analyses. Community Dent Oral Epidemiol. 2018;46(2):118-24.

26. Rogers HJ, Rodd HD, Vermaire JH, Stevens K, Knapp R, El Yousfi S, et al. A systematic review of the quality and scope of economic evaluations in child oral health research. BMC Oral Health. 2019;19(1):132.
27. Drummond MF, Jefferson TO. Guidelines for authors and peer reviewers of economic submissions to the BMJ. BMJ. 1996;313(7052):275-83.

28. Higgins JPT, Thomas J. Cochrane handbook for systematic reviews of interventions. Higgins JPT, Green S, editors. The Cochrane Collaboration; 2020.

29. Husereau D, Drummond M, Petrou S, Carswell C, Moher D, Greenberg D, et al. Consolidated Health Economic Evaluation Reporting Standards (CHEERS)-explanation and elaboration: a report of the ISPOR Health Economic Evaluation Publication Guidelines Good Reporting Practices Task Force. Value Health J Int Soc Pharmacoecon Outcomes Res. 2013;16(2):231-50.

30. Landis JR, Koch GG. The measurement of observer agreement for categorical data. Biometrics. 1977;33(1):159-74.

31. Caro JJ, Briggs AH, Siebert U, Kuntz KM, ISPOR-SMDM Modeling Good Research Practices Task Force. Modeling good research practices-overview: a report of the ISPOR-SMDM Modeling Good Research Practices Task Force-1. Value Health J Int Soc Pharmacoecon Outcomes Res. 2012;15(6):796-803.

32. Torgerson DJ, Raftery J. Discounting. BMJ. 1999;319(7214):914-5.

33. Roberts M, Russell LB, Paltiel AD, Chambers M, McEwan P, Krahn M, et al. Conceptualizing a model: a report of the ISPOR-SMDM modeling good research practices task force-2. Value Health. 2012;15:804-11.

34. Briggs $A H$, Weinstein MC, Fenwick EAL, Karnon J, Sculpher MJ, Paltiel AD. Model Parameter Estimation And Uncertainty Analysis: A Report Of The ISPOR-SMDM modeling good research practices task force working group-6. Med Decis Making. 2012;32(5):722-32.

35. Barbieri M, Drummond MF. Conflict of interest in industry sponsored economic evaluations: Real or imagined? Curr Oncol Rep. 2001;3(5):410-3.

36. NICE. Guide to the methods of technology appraisal 2013 [Internet]. 2013 [cited 2019 Dec 19]. https://www.nice.org.uk/process/pmg9/chapter/ foreword

37. Foster Page LA, Beckett DM, Cameron CM, Thomson WM. Can the Child Health Utility 9D measure be useful in oral health research? Int J Paediatr Dent. 2015;25:349-57.

38. Rogers HJ, Gilchrist F, Marshman Z, Rodd HD, Rowen D. Selection and validation of a classification system for a child-centred preference-based measure of oral health-related quality of life specific to dental caries. J Patient-Rep Outcomes. 2020;4(1):105.

39. Hettiarachchi R, Kularatna S, Byrnes J, Mulhern B, Chen G, Scuffham PA. Valuation study for a preference-based quality of life measure for dental caries (Dental Caries Utility Index-DCUI) among Australian adolescents—study protocol. BMJ Open. 2020;10(10):e038626.

\section{Publisher's Note}

Springer Nature remains neutral with regard to jurisdictional claims in published maps and institutional affiliations.
Ready to submit your research? Choose BMC and benefit from:

- fast, convenient online submission

- thorough peer review by experienced researchers in your field

- rapid publication on acceptance

- support for research data, including large and complex data types

- gold Open Access which fosters wider collaboration and increased citations

- maximum visibility for your research: over 100M website views per year

At BMC, research is always in progress.

Learn more biomedcentral.com/submissions 